\title{
Analysis of the optical transmission through a nonlinear thin layer near the critical angle of incidence
}

\section{R. Chifahi}

\section{R. Mountasser}

mountasser@yahoo.com

\section{A. Taouri}

\begin{abstract}
Université Hassan II Mohammedia, Faculté des Sciences et Techniques, Laboratoire d'Optoélectronique, Analyse optique et simulation, BP 146, Mohammedia, Maroc

Université Hassan II Mohammedia, Faculté des Sciences et Techniques, Laboratoire d'Optoélectronique, Analyse optique et simulation, BP 146, Mohammedia, Maroc
\end{abstract}

Université Hassan II Mohammedia, Faculté des Sciences et Techniques, Laboratoire d'Optoélectronique, Analyse optique et simulation, BP 146, Mohammedia, Maroc

In this communication, we present an analytical study of the linear and nonlinear optical transmission through a thin layer near the critical angle of incidence. The optical linear transmission presents very important variations according to the optical Kerr effect. The study of the linear optical transmission allows defining the operation point of the thin layer in nonlinear regime. We have studied the nonlinear transmission for different operating points near the critical angle of incidence. These study effects are important for optical applications such as the all optical switching and the sensitive determination of the nonlinear refractive index $\left(n_{2}\right)$ of materials. [DOI: 10.2971/jeos.2011.11023]

Keywords: optical Kerr effect, nonlinear thin layer, all optical switching

\section{INTRODUCTION}

All optical components, such as, modulators, logic gates, commutators etc. are based on the nonlinear response of dielectric Kerr materials (optical media having an intensitydependent refractive index are susceptible to enhance considerably, the performances of ultrafast information treatment systems, particularly, in telecommunications).

The study of the nonlinear transmission near the critical angle of incidence through a nonlinear thin Kerr layer is of great importance in order to realise an all optical commutator [1]-[3]. Also, for its high sensibility near the critical angle of incidence, the nonlinear transmission can be used to determine the nonlinear refractive index $\left(n_{2}\right)$ of thin films.

In this communication, we present an analytical study of the optical transmission through a thin nonlinear layer (Kerr material) near the critical angle of incidence. The study is made for different operating points situated on the last peak of oscillation of the linear transmission of the thin film. This study allows optimising the operating mode of the thin layer to appropriate applications mentioned above. In this study, we consider the case of a thin layer without absorption.

\section{PRINCIPLE}

We consider an optical thin Kerr layer (Figure 1) with a thickness $e$ and an intensity-dependent refractive index, given by: $n=n_{0}+n_{2} l$, where $n_{0}$ and $n_{2}$ are respectively, the linear index and Kerr coefficient and I the local intensity. Two identical linear media whose index of refraction $n_{1}$ is

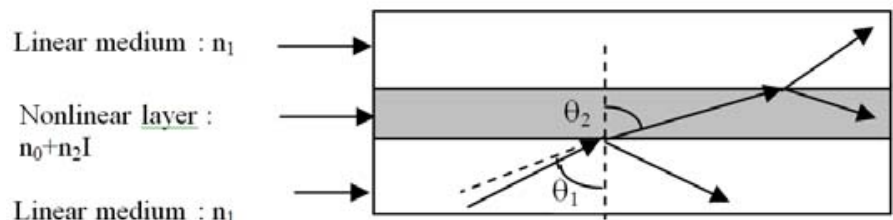

FIG. 1 Optical transmission through a nonlinear layer with thickness e and optical refractive index $\left(n=n_{0}+n_{2} I\right)$. The angle of incidence $\theta_{1}$, slightly lower than the critical angle of incidence $\theta_{c}=\arcsin \left(n_{0} / n_{1}\right)$.

slightly higher than $n_{0}$ surround the layer.

The layer is illuminated (Gaussian beam) under an angle of incidence $\theta_{1}$, slightly lower than the critical angle of incidence $\theta_{c}=\arcsin \left(n_{0} / n_{1}\right)$.

The nonlinear critical angle $\theta_{c}=\arcsin \left(\left(n=n_{0}+n_{2} l\right) / n_{1}\right)$ varies versus the optical Kerr term $n_{2} l$. It increases or decreases following that the Kerr coefficient is positive or negative. In the case where the incidence angle is close to the critical one, if we increase the intensity $I$, the optical transmission $T$ of the thin layer increases or decreases following that $n_{2}$ is positive or negative. This effect is the basis of all optical commutation by a nonlinear thin layer [1].

\section{LINEAR TRANSMISSION}

Experimentally, the incident light on the layer is a Gaussian 


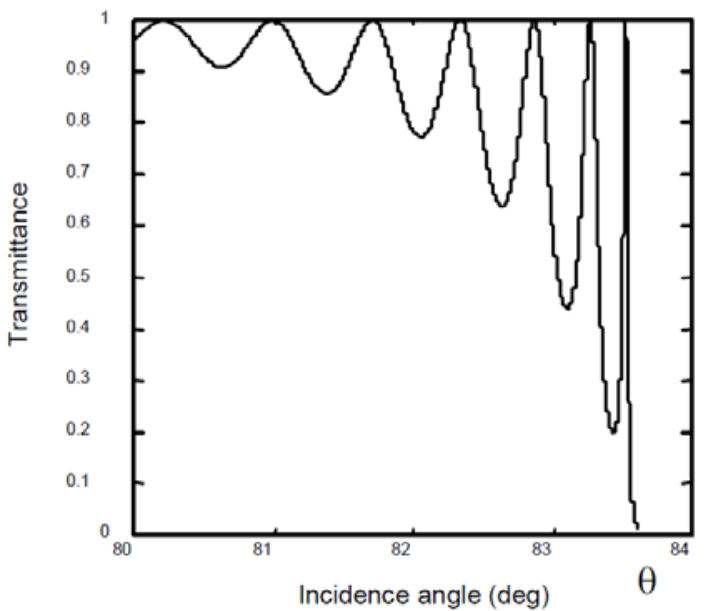

a)

FIG. 2a) Linear optical transmittance $\left(n_{2} I \sim 0\right)$ near the critical angle $\left(q_{c} \sim 83.61^{\circ}\right)$. b) Magnification of the last peak of $(T)$ in $\left.2 a\right)$.

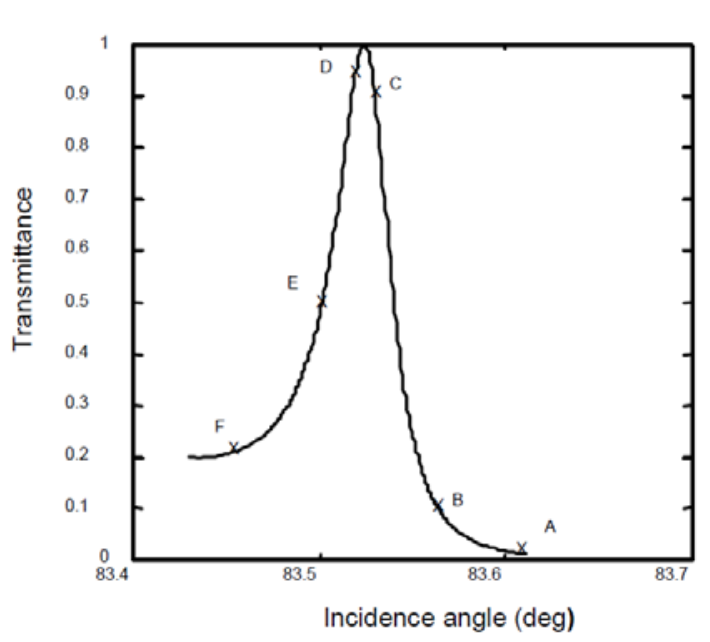

b) 

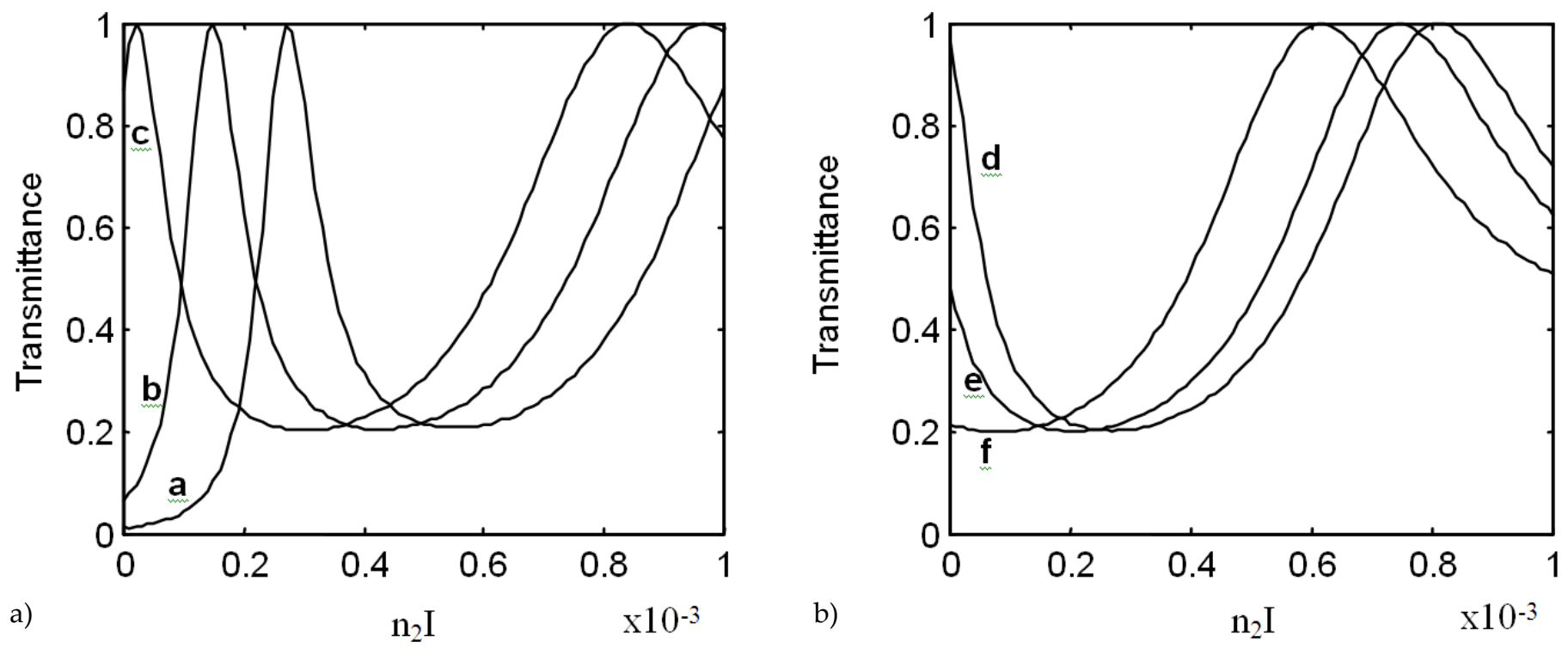

FIG. 3 Transmittance $T$ for a thin layer versus a positive Kerr effect $n_{2} I\left(n_{2}>0\right)$. The curves (a, b, c, d, e and f) correspond the operating points (A, B, C, D, E and F).

at the first order by [5]:

$$
\Delta \beta \approx\left(2 \pi / \lambda_{0}\right) \text { e } n_{2} I\left[1+\left(2 \Delta \theta \sqrt{2 \Delta n / n_{1}}\right)^{-1 / 2}\right]
$$

With $\mathrm{D} q=q_{\mathrm{c}}-q_{1}$ and $\mathrm{D} n=n_{1}-n_{0}$.

In the dephasing $\beta$, both $\mathrm{Dq}$ and $\mathrm{Dn}$ are very small and the second term of the sum in brackets is very important. It is more than ten times greater than the first term due to the physical parameters of the layer. This explain the high sensibility of the transmittance of a resonant thin layer near the critical angle. In order to show the high sensibility of the transmittance $(T)$ near the critical angle of incidence, we present a numerical study of the considered thin layer versus the nonlinear index $\left(n_{2} l\right)$. I is the local intensity inside the cavity. The variation of the transmittance $(T)$ versus the optical Kerr effect depends on the sign of $n_{2}$ and the position of the operating point $Q$. On the last peak, we study position of $Q$ in two situations. With increasing incident intensities, the variation of the transmittance depends on the sign of $n_{2}$.

\subsection{Positive $n_{2}$}

In this case, the transmittance varies as if the operating point is going toward the left. To study $(T)$ versus $n_{2} l$, we chose three operating points: $\mathrm{A}\left(\theta_{1}=83.61^{\circ}, \mathrm{T}=0.01\right)$; $\mathrm{B}\left(\theta_{1}=83.57^{\circ}\right.$, $\mathrm{T}=0.08) ; \mathrm{C}\left(\theta_{1}=83.53^{\circ}, \mathrm{T}=0.90\right)$ on the trailing edge and three others operating points $\mathrm{D}\left(\theta_{1}=83.52^{\circ}, \mathrm{T}=0.96\right)$; $\mathrm{E}\left(\theta_{1}=\right.$ $\left.83.50^{\circ}, \mathrm{T}=0.48\right) ; \mathrm{F}\left(\theta_{1}=83.46^{\circ}, \mathrm{T}=0.22\right)$ on the rising of the last oscillation peak. The transmittance is determined versus the optical Kerr effect by replacing the linear index $n_{0}$ in Eqs. (1)-(4) by the dependent-intensity index $\left(n_{0}+n_{2} l\right)$.

To apply rigorously the formulas indicated above, it is necessary to know the values of the local intensity I inside the cavity. According to the characteristics of the layer, the finess $F$ of the Fabry-Perot resonator considered is low:

$$
F=\pi(R)^{1 / 2} /(1-R) \sim 3.7
$$

Thus the local intensity I can be estimated by an average value $I$ [7].

$$
I \approx \frac{(1+R)}{(1-R)} I_{t}
$$

$R$ is the reflectance of the layer: $R=\left|r_{1}\right|^{2}$, and $I_{t}$ the transmitted intensity: $I_{t}=T \times I_{0}$

So, the Eqs. (1)-(7) allow us to study the transmittance $T$ versus the incident intensity $I_{0}$. To avoid specifying the value of $\mathrm{n}_{2}$, we have represented $T$ versus term of global Kerr $n_{2} l$.

The results of the analytical study of the transmittance $(T)$ versus $n_{2} l$ are shown in Figure 3.

In Figure 3a, the separated curves $a, b$, and c correspond to the operating points $\mathrm{A}, \mathrm{B}$, and $\mathrm{C}$ while the others separated curves $d, e$, and $f$ in Figure $3 b$ correspond to the operating points $\mathrm{D}, \mathrm{E}$, and $\mathrm{F}$. We note that a very important result is shown in Figure 3: When the incident intensity rises (from zero), the resulting transmittance can either increase or decrease depending on the position of the operating point. Also, we observe in Figure $3 a$ that as the intensity increases from near zero, the corresponding transmittance increases monotonaly and this increase becomes more and more important as the incidence angle approaches the critical angle. So, when the term $n_{2} l$ increases from 0 , the transmittance $T$ increases in a monotone fashion for: the operating point $\mathrm{A}$ from 0 to 1,for the point $B$ from 0.08 to 1, and for the point $C$ from 0.9 to 1 .

All these results show the importance to take an operating point near the angle of total reflection.

For the operating points (D, E, and F) taking on the ascendant slope of the last oscillation peak, it is shown in Figure $3 \mathrm{~b}$ that by increasing the optical Kerr effect, progressively, from zero, the transmittance decreases with a monotone rate which becomes (this rate) more and more important as these operating points approaches the summit $(\mathrm{F} \rightarrow \mathrm{E} \rightarrow \mathrm{D})$. 
From a commutation point of view, the Figure 3a shows in an evident way the commutation effect from a low level (0) to a high level (1) as the incident intensity is increased. In reverse, Figure $3 b$ shows the commutation effect from a high level (1) to low level (0).

\subsection{Negative $n_{2}$}

For a negative Kerr coefficient $n_{2}$, the results of the transmittance variation versus the nonlinear refraction index $n_{2} l$ are reversed to those obtained for $\mathrm{n}_{2}$ positive. If the operating point $(\mathrm{A}, \mathrm{B}$ or $\mathrm{C})$ is taken on the descendent slope, the increase of the incident intensity trains a reduction of the transmittance. This reduction is affected in a monotone and significant fashion as the operating point approaches the summit $(\mathrm{A} \rightarrow \mathrm{B} \rightarrow \mathrm{C})$. Otherwise, if the operating point is on the ascendant slope, an increase of the transmittance is observed with increasing incident intensity.

\section{CONCLUSION}

In this paper, we have presented an analytical study of the transmittance through a thin layer near the critical angle of incidence. We have analysed the transmittance of a thin layer versus the incidence angle and the optical Kerr term $n_{2} l$. This study has allowed us to show the high sensibility of the transmittance near the total reflection in resonant regime. For different operating points, we have studied the nonlinear transmittance versus incident intensity in the resonant regime. The two cases where the Kerr effect is positive or negative are studied, too. This study has allowed us to optimize the use of a nonlinear thin film for the applications to all optical switching and to the determination of $n_{2}$.

\section{References}

[1] A. E. Kaplan, "Hysteresis reflection and refraction by a nonlinear boundary" JETP Lett. 24, 114-119 (1976).

[2] E Lantz, D. Métin, H. Cornet, and A. Lacourt, "Transmission of a gaussian beam through a nonlinear thin film near the total reflection state" J. Opt. Soc. Am. B 11, 347-354 (1994).

[3] R. Mountasser, and A. Lacourt, "Réfraction non linéaire au voisinage de l'angle limite" Ann. Phys-Paris 20, 93-95 (1995).

[4] R. Mountasser, H. Maillotte, F. Cherioux, and M. Ayadi, "Picosecond optical limiting action through a thin MMA-optopole copolymer layer" Nonlinear Optic 25 (2000), p. 373-378.

[5] R. Mountasser, E. Lantz, and H. Maillotte, "Transmission through a nonlinear thin layer near the critical angle of incidence: application to the sensitive determination of the nonlinear refractive index" J. Opt. A-Pure Appl. Op. 4, 303-308 (2002).

[6] M. Born, and E. Wolf, Principles of optics (6th Edition, Pergamon Press, Oxford, 1980).

[7] M. Haelterman, G. Vitrant, and R. Reinisch, "Transverse effects in nonlinear planar resonators: I. Modal theory" J. Opt. Soc. Am. B 7, 1309-1318 (1990). 